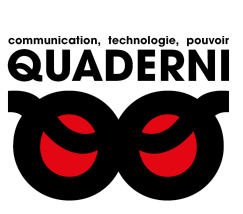

\title{
Quaderni
}

Communication, technologies, pouvoir

95 | Hiver 2017-2018

Logiques numériques des radicalisations

\section{Aurore Van de Winkel, Les légendes urbaines de Belgique}

\section{Véronique Campion-Vincent}

\section{(2) OpenEdition}

10 Journals

\section{Édition électronique}

URL : https://journals.openedition.org/quaderni/1151

DOI : 10.4000/quaderni. 1151

ISSN : 2105-2956

Éditeur

Les éditions de la Maison des sciences de l'Homme

\section{Édition imprimée}

Date de publication : 5 février 2018

Pagination : 121-126

\section{Référence électronique}

Véronique Campion-Vincent, "Aurore Van de Winkel, Les légendes urbaines de Belgique », Quaderni [En ligne], 95 | Hiver 2017-2018, mis en ligne le 05 février 2018, consulté le 04 septembre 2021. URL:

http://journals.openedition.org/quaderni/1151; DOI : https://doi.org/10.4000/quaderni.1151 


\section{Compte rendu}

\section{Les légendes urbaines de Belgique}

Aurore Van de Winkel

Waterloo, Avant-propos, 2017

par Véronique Campion-Vincent

Attachée scientifique

Fondation Maison des Sciences de l'Homme

C'est un ouvrage fort distrayant, mais également bourré d'informations des plus sérieuses, qu'a réalisé Aurore Van de Winkel avec Les légendes urbaines de Belgique.

Dans le champ des rumeurs et légendes urbaines, celle-ci mène de front recherches et interventions (www.grezlesrumeurs.com), opérant en Belgique comme en France. Chargée de cours à l'Université St Louis de Bruxelles et à l'IUT de Troyes, elle est titulaire d'un doctorat en Sciences de l'Information et de la Communication de l'Université de Louvain (2009).

Cet ouvrage fait suite à Gérer les rumeurs, ragots et autres bruits (Edipro, 2012) davantage tourné vers les interventions.

Les rumeurs et légendes urbaines ont vu leur importance croître en raison de leur emploi massif des nouvelles techniques de l'information. Cet ouvrage se situe donc dans le champ de Quaderni car les technologies nouvelles ont considérablement accru l'importance de ces communications qui mettent en cause les aspects officiels de nos sociétés.

Les sept parties de la table des matières, «Aliments suspects », « Femmes victimes », «Animaux déplacés », « Enfants en danger », « Agressions tous azimuts », « Astuces et revanches », « Signes à décrypter », guident le lecteur dans une promenade plaisante parmi les variantes des légendes urbaines, récits qui dénoncent et commentent avec force détails saisissants les horreurs du 
monde actuel. Aurore Van de Winkel a fort bien su repérer l'essentiel, qui au-delà de leur apparition et évolution se niche dans les messages implicites transmis par ces histoires car «les légendes urbaines sont destinées à être crues » (p. 17). S'appuyant sur ses expériences participatives de promenades légendaires dans les rues de Bruxelles autant que sur sa réelle érudition et son active fréquentation du net Aurore Van de Winkel dresse ici un panorama complet des légendes urbaines actives en Belgique.

La plume est plaisante, et étudie aussi très sérieusement sans aucun pédantisme les conditions sociales conflictuelles, les débats historiques tout comme les légendes traditionnelles dans lesquelles s'enracinent les légendes urbaines racontées aujourd'hui.

On présentera ici en détail deux chapitres, l'un touchant aux peurs élémentaires universelles l'autre regroupant des inquiétudes plus sophistiquées d'un public averti, inquiétudes liées au décodage généralisé contemporain. Cette présentation permettra de saisir l'originalité de la trame narrative adoptée par l'auteur. Ainsi « Des aliments suspects » relate successivement :

Une anecdote de contamination : " elle commanda une pita [...] quelques heures plus tard [...] ses amis furent obligés d'appeler une ambulance ». Après un lavage d'estomac «ils découvrirent, non seulement les restes de la pita mais également six sortes de spermes différents » (p. 20). Puis des variantes : «dans la célèbre discothèque Le Carré à Willebroek la nourriture contiendrait non seulement de la semence humaine mais également du sang menstruel! »; à Paris « un employé du Burger King, accusé du même méfait, est également séropositif». Et un démenti suivi d'un rapide décodage : «Cette légende qui ne met guère en appétit, ne repose bien sûr pas sur des faits. [...] Le récit reflète plutôt la peur du manque d'hygiène [...] et la suspicion envers l'utilisation assez généralisée de nourriture industrielle et bon marché » (p. 21) dont le consommateur ne peut surveiller la fabrication.

Puis plusieurs contaminations proches sont évoquées : traces d'urine dans les ramequins de cacahuètes offerts dans les cafés et bars, ingrédients divers et peu ragoutants (globes oculaires bovins, œufs d'araignées, vers de terre, mais aussi chameau, cheval, chat ou rat) dans les steaks hachés de McDonald et autres fast-food. Une variante inédite (à son maximum en 2007-2008) affirme qu'une petite pastille anti-vomitive est visible sur le pain des hamburgers de 
McDonald ou de Quick : elle est insérée par les entreprises afin que le client ne vomisse qu'une fois sorti du restaurant (p. 23).

Après une analyse du contexte de ces récits : fraudes alimentaires, vrais scandales (de la vache folle, du poulet à la dioxine, etc..), néophobie, historique des peurs alimentaires à l'ère préindustrielle (p. 23-25), c'est le thème du cannibalisme involontaire qui est abordé et les récits inquiétants sont traités de concert avec les récits à la Sweeney Todd de barbiers meurtriers complices de charcutiers peu scrupuleux proposant de délicieux pâtés, sans oublier le boucher meurtrier dont les victimes sont ressuscitées par St Nicolas dans la chanson populaire d'autrefois (p. 25-30).

Le thème des fraudes alimentaires réelles observées en Belgique, particulièrement en période de pénurie lors des guerres mondiales (p. 31-32), conduit ensuite à la rumeur selon laquelle les industriels vendaient des calamars frits composés d'anus de cochon (p. 33-35). Cette histoire a eu droit à une validation exceptionnelle par une eurodéputée néerlandaise qui la relata à des journalistes pour souligner l'intérêt d'un rapport sur la fraude alimentaire qu'elle venait de remettre au Parlement européen (17 octobre 2013). Le fait est relaté avec précision en notant la reprise sans vérification par la presse francophone, puis la reprise de la rumeur par les réseaux sociaux où elle circule toujours en dépit des démentis de presse qui suivirent. Il ne s'agit là que d'une réédition car la rumeur, notée aux États-Unis en 2010, y réapparut lors de la sortie d'une législation sur la sécurité alimentaire. Son utilité - elle pose un politicien qui veut intéresser des journalistes et souligner la nécessité de législation et de contrôle - mais également son absurdité sont soulignées par l'auteur : « Si un calamar et son encornet peuvent donner au fabricant un certain nombre de rondelles, n'oubliez pas que chaque cochon n'a qu'un anus ! [...] il deviendrait fastidieux et économiquement peu rentable d'en récolter assez pour remplir nos assiettes aussi copieusement que peut le faire un calamar » (p. 35).

Les récits et thèmes suivants nous parlent de l'os de rat (ingéré dans un plat savouré au restaurant asiatique) (p. 35-36), des rats « officiels » au restaurant (certains établissements à Bruxelles servaient du ragondin en spécialité) (p. 37-38), du chien familier amené en plat à ses maîtres par des employés chinois désireux de bien faire (p. 38), des chats proposés en guise de poulets ou lapins (p. 39-40, 43), des pratiques réelles de consommation de chien en 
Belgique (p. 40-42). Après l'alerte apparue en 2000 aux bananes tueuses $d u$ Costa Rica soi-disant porteuses de la terrible « fasciite nécrosante » mangeuse de chair (il s'agit d'une bactérie réelle mais sans lien avec l'ingestion d'aliments) (p. 44-47) on passe aux boissons : Red Bull et taurine (prise à tort pour un produit d'origine animale) (p. 48-49), le mélange explosif Mentos/Coca très populaire sur YouTube (p. 50-51), le Coca (p. 51-53), les canettes contaminées à la Leptospirose (p. 54-55), les accusations anciennes de profanation d'hosties par des juifs (p. 55-56 en note) et modernes d'empoisonnement des eaux par l'ennemi (p. 55-56). La mise en cause de Maggi et du bouillon Kub espions et auxiliaires des Allemands selon la droite française (p. 57-59) conduit aux accusations multiples de bonbons empoisonnés (p. 59-66). Après un détour par le tract, dit de Villejuif car attribué à un hôpital connu, mettant en cause les additifs alimentaires (apparu en 1976 en France, relancé en 2009 contre la Commission Européenne siégeant à Bruxelles) tract qui a connu la gloire d'être cité par un manuel scolaire français comme authentique et est toujours bien vivant (p. 66-70) le chapitre se clôt sur le Strawberry Quick (ou Strawberry Meth), une nouvelle drogue qui se loverait dans des bonbons pétillants (p. 70-73).

Le dernier chapitre « Décryptez les Signes » passe en revue Les chaussures suspendues (p. 232-235) et les diverses hypothèses que suscite leur présence. Les signes des cambrioleurs sur nos maisons (p. 235-247) avec reproduction de nombreuses listes circulant souvent avec l'aval d'autorités locales. Le langage secret des publicités - auxiliaires des envahisseurs allemands en Belgique - citant de nombreux articles de presse de l'époque et fort bien illustré (p. 247-252) puis de nos jours : Marlboro et signes d'appartenance au Ku Klux Klan, « Chokotoffs » et papier d'emballage spécial porteur de récompenses, drapeau européen inspiré de la médaille miraculeuse de la Vierge apparue rue du Bac à Paris (p. 252-254). Auto-stoppeurs fantômes et dames blanches (p. 255-264). Prédictions de catastrophes et fins du monde (comètes 1726, 1857, 1910, an 2000, 21 décembre 2012) (p. 264-276).

Cet ouvrage se distingue des autres anthologies de légendes urbaines par l'attention portée à la présence locale des légendes.

La présentation de l'ouvrage est son point fort car il comporte une très riche iconographie fort bien mise en page. Cette iconographie présente un ancrage géographique prononcé : de nombreuses illustrations (les photographies, 
excellentes, œuvre du père de l'auteur, situent les lieux où les histoires se déroulent (se dérouleraient?).

Dans les textes consacrés à l'exposé d'un récit, son voyage et ses mutations d'une localité à l'autre sont longuement détaillés.

L'ouvrage offre des exemples multiples des diverses incarnations des légendes urbaines relatées : les textes des messages internet sont reproduits sur des fonds colorés mais on trouve aussi des coupures de presse, des tracts, des illustrations. Beaucoup d'exemples sont tirés de Bandes Dessinées, une forme d'expression artistique particulièrement développée en Belgique et dont les auteurs ont fréquemment illustré des thèmes de légendes urbaines.

Les textes illustrant le climat social dans lequel se développent les légendes urbaines sont très largement d'origine belge : presse, mais aussi recueils d'histoire locale et de textes folkloriques, souvent du XIX ${ }^{\mathrm{e}}$ siècle, qui permettent de faire le lien avec les légendes du passé.

La bibliographie, précise, est très développée et clairement indiquée. Les ouvrages de commentaires sont distingués des « sources primaires » relatant des légendes.

Le lecteur pointilleux regrettera l'absence d'un index qui aurait fait de ce bel ouvrage un précieux instrument de référence. Il s'agit davantage d'un ouvrage grand public que d'une étude scientifique, cependant c'est une lecture que l'on doit recommander à tous ceux que les créations culturelles collectives contemporaines intéressent. 
